\section{Concomitant WT1 mutations predict poor prognosis in acute myeloid leukemia patients with double mutant CEBPA}

Acute myeloid leukemia (AML) with double mutant CCAAT/enhancer binding protein $\alpha\left(C E B P A^{\mathrm{dm}}\right)$ is a new entity in the 2016 World Health Organization (WHO) classification with unique biologic features and prognostic implications. ${ }^{1,2}$ The incidence of $C E B P A^{\mathrm{dm}}$ ranges from $7.5 \%$ to $11 \%$ in AML. ${ }^{1,3,4} C E B P A^{\mathrm{dm}}$ AML patients, when treated with standard chemotherapy, achieve a high complete remission (CR) rate. However, relapse occurs in $40 \%$ of patients who attain CR. ${ }^{1}$ This has raised the clinically relevant question whether concomitant genetic alterations influence the prognosis of $C E B P A^{\mathrm{dm}}$ patients. Apart from GATA2, the prognostic impact of other concomitant gene mutations is largely unsettled because limited patient numbers preclude informative analyses. ${ }^{5}$ Given that AML is a heterogeneous disease, risk-adapted treatment may not only improve the prognosis, but also reduce toxicity from the therapy. Allogeneic hematopoietic stem cell transplantation (allo-HSCT) in first CR is not beneficial for cytogenetically normal AML (CN-AML) patients with $C E B P A^{\mathrm{dm}} .^{6}$ If any concomitant mutations adversely affect the clinical outcome of $C E B P A^{\mathrm{dm}}$ patients, it will be interesting to know whether allo-HSCT should be performed for these patients. As yet, there is no data to answer this question.

In this study, the aim was to identify additional mutations in $C E B P A^{\mathrm{dm}}$ AML patients that conferred prognostic significance. Furthermore, we investigated the role of allo-HSCT in CEBPA ${ }^{\mathrm{dm}}$ patients with concurrent adverserisk mutations. Mutation analyses in CEBPA and 19 other relevant genes, including FLT3-ITD, FLT3-TKD, NRAS, KRAS, KIT, PTPN11, RUNX1, GATA2, MLL/PTD, ASXL1, IDH1, IDH2, TET2, DNMT3A, SF3B1, SRSF2, U2AF1, NPM1, WT1, and TP53 were performed by Sanger sequencing for patients $(n=500)$ diagnosed from 1994 to $2007 .{ }^{7,8}$ For patients $(n=256)$ diagnosed after 2008, Ion Torrent next generation sequencing (NGS) (Thermo Fisher Scientific, MA, USA) was performed. The WT1 mutations detected by NGS were all confirmed by Sanger sequencing.

We identified $102(13.5 \%)$ CEBPA-mutated patients from 756 patients with newly diagnosed de novo AML (Online Supplementary Table S1); 33 (4.4\%) had CEBPA single mutation $\left(C E B P A^{\mathrm{sm}}\right)$ and $69(9.1 \%), C E B P A^{\mathrm{dm}}$. Sixtynine $C E B P A^{\mathrm{dm}}$ patients were found to have 109 distinct mutations (Figure 1A, Online Supplementary Table S2). All patients had a combination of one $\mathrm{N}$-terminal and one $C$-terminal mutation. Most (53 of $56,94.6 \%$ ) of the $\mathrm{N}$-terminal mutations were frame-shift mutations, while most (42 of $53,79.2 \%$ ) of the $C$-terminal mutations were in-frame mutations with internal tandem duplications clustered in the junction between the basic region and the leucine zipper.

$C E B P A^{\mathrm{dm}}$ patients were significantly younger and had higher hemoglobin levels at diagnosis than $C E B P A^{\mathrm{sm}}$ and $C E B P A$ wild-type patients. All except one $C E B P A^{\mathrm{dm}}$ patient had intermediate-risk cytogenetics $(P<0.0001)$ (Figure 1A). The most frequent intermediate-risk cytogenetic change was $\operatorname{del}(9)(n=4,5.8 \%)$, and $\mathrm{CN}-\mathrm{AML}$ occurred in $81.2 \%$ of $C E B P A^{\mathrm{dm}}$ patients $(\mathrm{n}=56)$.

Fifty $(72.5 \%)$ of the $C E B P A^{\mathrm{dm}}$ patients had additional genetic alterations (Online Supplementary Table S3). Among them, 29 (58\%) had one, 17 (34\%) had two, 3 $(6 \%)$ had three and $1(2 \%)$ had four changes. The most common concurrent molecular event in CEBPA $A^{\mathrm{dm}}$ patients was GATA2 mutation (33.8\%), followed by FLT3-ITD $(14.5 \%)$, NRAS $(14.5 \%)$, TET2 $(13.2 \%)$, and WT1 $(11.8 \%)$ mutations. GATA2 was more frequently mutated in $C E B P A^{\mathrm{dm}}$ patients than in CEBPA wild-type patients $(33.8 \%$ vs. $2.8 \%, P<0.0001)$. In contrast, $C E B P A^{\mathrm{dm}}$ patients less frequently harbored NPM1, ASXL1, IDH2, $D N M T 3 A$ and $R U N X 1$ mutations (Figure 1B).

Survival analyses were restricted to 530 patients, including $62 C E B P A^{\mathrm{dm}}$ patients and 468 others (22 with $C E B P A^{\text {sm }}$ and $446 C E B P A$ wild-type), who received standard intensive chemotherapy. The CR rate was $90.2 \%$ for $C E B P A^{\mathrm{dm}}$ patients and $72.2 \%$ for others $(P=0.003)$. In multivariate analysis, $C E B P A^{\mathrm{dm}}$ was an independent favorable prognostic factor for OS and DFS (RR 0.420, 95\% CI $0.246-0.718, P=0.002$ and RR $0.544,95 \%$ CI 0.351-0.842, $P=0.006$, respectively, Online Supplementary Table S4). Of the $56 C E B P A^{\mathrm{dm}}$ patients who achieved first CR, 10 received allo-HSCT and 46 had postremission chemotherapy alone. The reasons for frontline alloHSCT were persistent residual leukemia cells in 4 patients, concurrent FLT3-ITD in 3 patients, initial hyperleukocytosis in 2 patients and complex cytogenetics in 1 patient. Intriguingly, the relapse rate was $45.7 \%$ in the postremission chemotherapy group and $0 \%$ in the alloHSCT group $(P=0.009)$. DFS was significantly better in

Table 1. Clinical characteristics and treatment outcome of CEBPA ${ }^{\mathrm{dm}}$ patients with concomitant WT1 mutations.

\begin{tabular}{|c|c|c|c|c|c|c|c|c|c|}
\hline JPN & Age/Sex & $\begin{array}{l}\text { WBC } \\
(\mathrm{k} / \mathrm{uL})\end{array}$ & Karyotype & $\begin{array}{l}\text { WI mutation } \\
\text { aa change }\end{array}$ & $\begin{array}{c}\text { Other } \\
\text { mutations }\end{array}$ & $\begin{array}{l}\text { Induction } \\
\text { response }\end{array}$ & Relapse & $\begin{array}{l}\text { Remission } \\
\text { duration } \\
\text { (months) }\end{array}$ & Outcome \\
\hline 24 & $55 \mathrm{M}$ & 58.2 & $\mathrm{CN}$ & P355C & - & CR1 & + & 9 & HSCT at CR2, alive \\
\hline 29 & $53 \mathrm{~F}$ & 94.6 & $+21,-x$ & D377fsX384 & - & CR1 & + & 11 & death \\
\hline 32 & $45 \mathrm{~F}$ & 3.3 & Complex & Y402X & - & CR1 & - & 91 & HSCT at CR1, alive \\
\hline 50 & $59 \mathrm{M}$ & 387.4 & $\mathrm{CN}$ & R369G & - & CR1 & + & 7 & death \\
\hline 54 & $40 \mathrm{M}$ & 160.0 & $\mathrm{CN}$ & K399fsX448 & - & Refractory & NA & 0 & death \\
\hline 56 & $35 \mathrm{M}$ & 248.0 & $\mathrm{CN}$ & R458X & FLT3-TKD & CR1 & + & 7 & HSCT at PR2, alive \\
\hline 62 & $28 \mathrm{~F}$ & 17.0 & $\mathrm{CN}$ & K399fsX400 & - & Refractory & NA & 0 & death \\
\hline 27 & $69 \mathrm{M}$ & 227.7 & NM & N381fsX450 & TET2 & $N A^{a}$ & NA & NA & NA \\
\hline
\end{tabular}

aa: amino acid; CN: cytogenetically normal; CR: complete remission; NA: not applicable; NM: no mitosis; HSCT: hematopoietic stem cell transplantation; PR: partial response; UPN: unique patient number. a ${ }^{\text {UPN27 }} 7$ lost to follow up after diagnosis. 
the allo-HSCT group (median, not reached (NR) vs. 59.4 months, $P=0.023$ ) than in the chemotherapy group, while OS was not different $(P=0.247)$ (Online Supplementary Figure S1).

We further analysed the prognostic significance of concomitant gene mutations with a frequency above $10 \%$ in $C E B P A^{\mathrm{dm}}$ patients. WT1-mutated patients tended to have a lower CR rate $(71.4 \%$ vs. $92.5 \%, P=0.14)$ and a higher relapse rate $(80 \%$ vs. $34 \%, P=0.047)$ compared to those with wild-type WT1 (Online Supplementary Table S5). With a median follow up of 69.7 months (range, 1.2-230 months), WT1-mutated patients had a significantly shorter OS and DFS than WT1-wild patients (median, 14 months vs. NR, $P=0.021 ; 7.8$ months vs. NR, $P=0.008$, respectively; Figure 1C). According to the 2017 European LeukemiaNet (ELN) classification, the AML patients were stratified into three risk groups (Figure 2A). Integration of WT1 mutations could further divide the ELN favorablerisk cohort into three subgroups: $C E B P A^{\mathrm{dm}} W T 1$-mutated patients, $C E B P A^{\mathrm{dm}} W T 1$-wild patients and others. As shown in Figure 2B, CEBPA ${ }^{\mathrm{dm}}$ patients with WT1 muta- tions had worse outcome than other ELN favorable-risk patients. Sequential analyses of WT1 mutations revealed that the mutations in three WT1-mutated patients in the study were lost at CR, but regained at relapse. The mutation burden could either increase or decrease at relapse. Of the 116 WT1-wild patients studied, three acquired a novel mutation at relapse (Online Supplementary Table S6).

Regarding other concomitant gene mutations, GATA2 mutation was correlated with a trend of longer DFS (median, NR vs. 16.1 months, $P=0.078$ ). FLT3-ITD, NRAS and TET2 mutations seemed not to have implications on the clinical outcome (Online Supplementary Table S7 and Online Supplementary Figure S2).

To our knowledge, this is the first study to demonstrate the prognostic impact of concurrent WT1 mutations on $C E B P A^{\mathrm{dm}}$ patients. WT1 mutation occurs in $6-10 \%$ of AML patients and is associated with poor prognosis in $\mathrm{CN}-\mathrm{AML}$ and non-selective AML patients. ${ }^{9,10}$ Intriguingly, WT1 mutations are frequently identified in CEBPA ${ }^{\mathrm{dm}}$ patients. ${ }^{11}$ We distinctly found that WT1 mutations were associated with poor clinical outcome in $C E B P A^{\mathrm{dm}}$

A

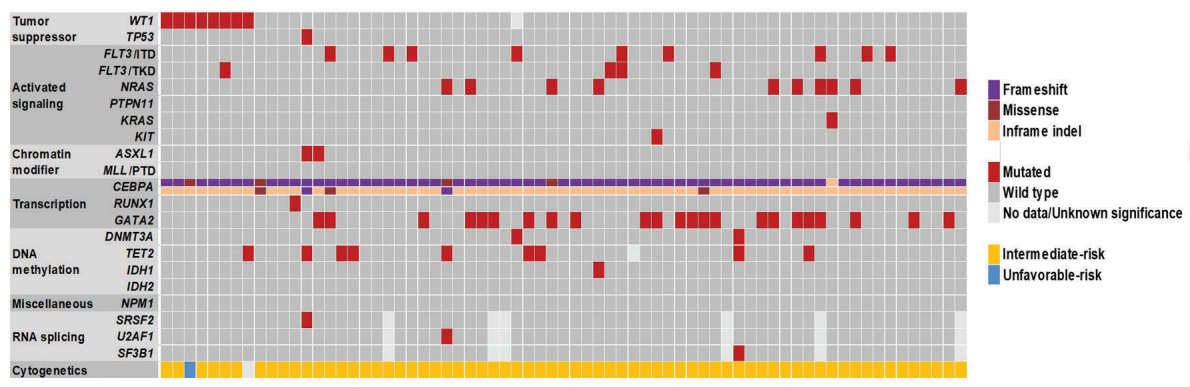

B

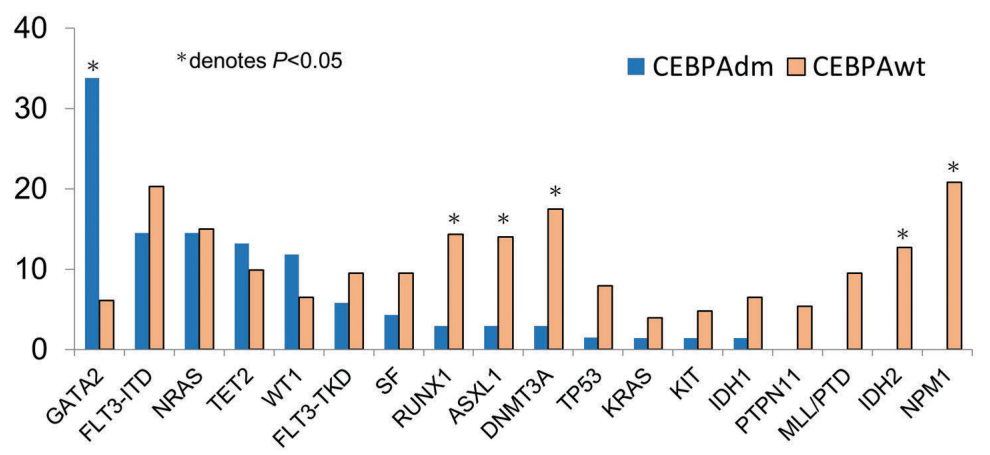

C
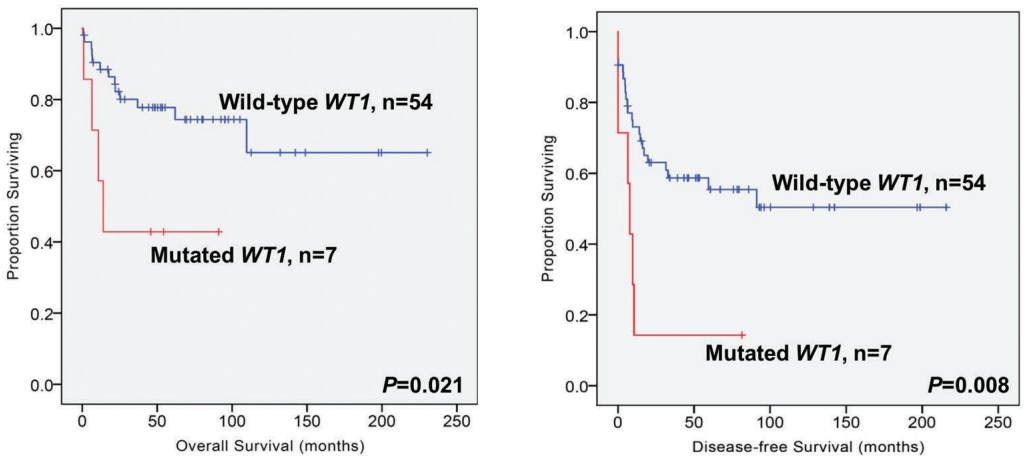

Figure 1. CEBPA double mutations $\left(C E B P A^{\mathrm{dm}}\right)$ in de novo AML patients. (A) The diagram of concurrent mutations in patients with CEBPA ${ }^{\mathrm{dm}}$. (B) The distribution of concomitant mutations in AML patients with either CEBPA ${ }^{\mathrm{dm}}$ or wild-type CEBPA. (C) Kaplan-Meier plots for OS (left) and DFS (right) according to WT1 mutation status in CEBPA ${ }^{\mathrm{dm}}$ patients. 
A

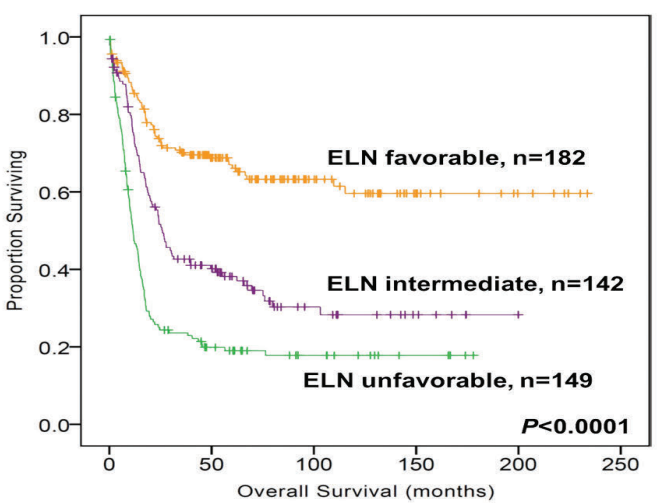

B

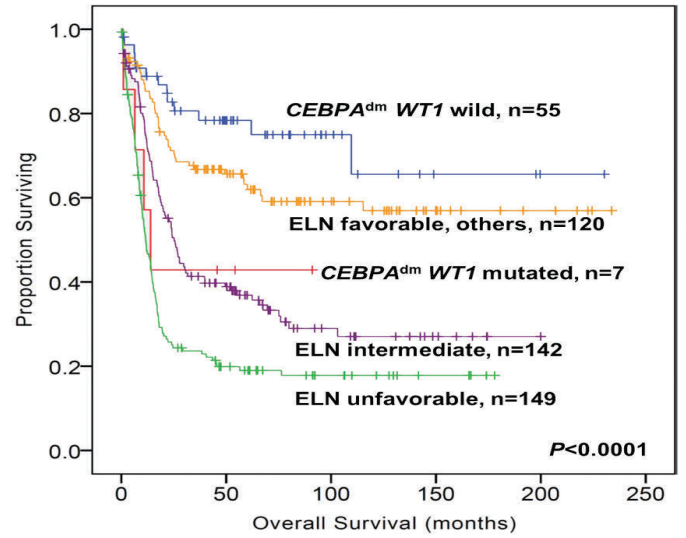

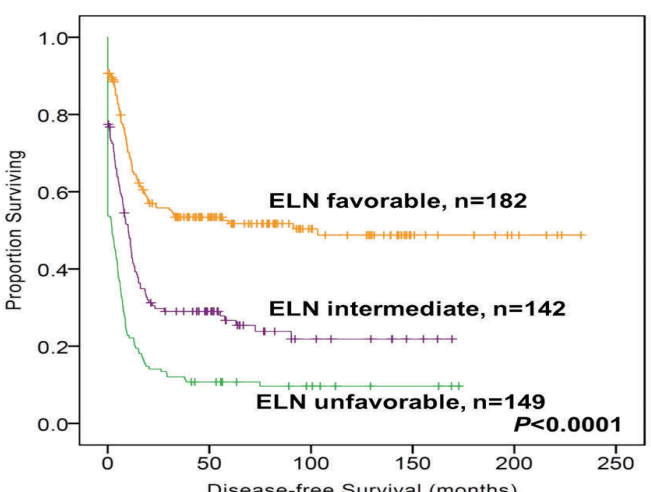

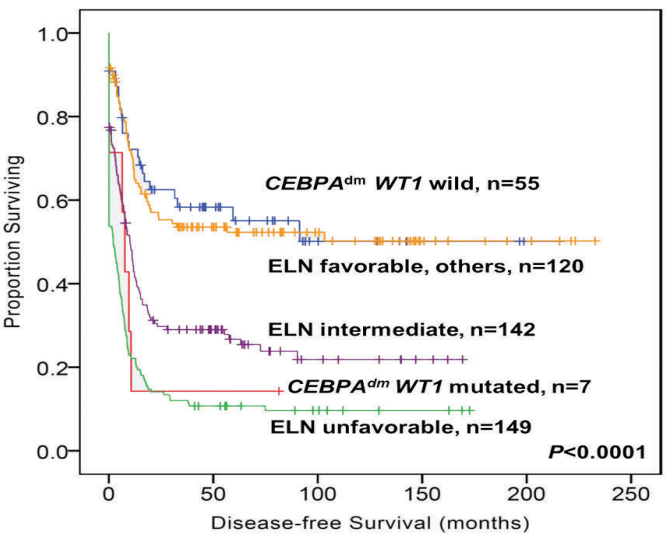

Figure 2. Risk stratification of the ELN favorable group according to the status of CEBPA and WT1 mutations. (A) Kaplan-Meier plots for OS and DFS stratified by the 2017 ELN risk categories. (B) ELN favorable group could be further separated into three subgroups according to the status of CEBPA and WT1 mutations. CEBPA ${ }^{\mathrm{dm}}$ patients with concurrent WT1 mutations had OS and DFS poorer than other ELN favorable-risk patients, but similar to those with the ELN intermediate (CR $76.8 \%$, relapse rate $56.0 \%$, median OS 26.0 months, median DFS 10.2 months) or unfavorable-risk category (CR $53.7 \%$, relapse rate $61.3 \%$, median 0S 11.6 months, median DFS 2.1 months). ELN:European LeukemiaNet.

patients. Furthermore, we showed that integration of WT1 mutations could refine the ELN risk stratification in favorable-risk subgroups. The prognostic impact of concomitant mutations in $C E B P A^{\mathrm{dm}}$ patients have not been widely assessed with the exception of GATA2 and TET2 mutations. ${ }^{3,5,12}$ Grossmann et al. showed that the presence of TET2 mutations correlated with worse survival. In contrast, we did not find the prognostic impact of TET2 mutations in $C E B P A^{\mathrm{dm}}$ patients.

A high frequency of TET2 co-mutation (around 34\%) in $C E B P A^{\mathrm{dm}}$ patients was reported previously, ${ }^{3,5}$ while it was only $13.2 \%$ in our study. The reason that our results were very different from those reported in other geographical areas might partly be explained by the difference in patient characteristics. The $C E B P A^{\mathrm{dm}}$ patients in the studies of Grossmann et al. ${ }^{5}$ and Fasan et al. ${ }^{3}$ were significantly older than ours (median age, 57.5 and $56.3 \mathrm{vs}$. 40 years). It is well documented that TET2 mutations occur more frequently in elderly AML patients than younger ones ${ }^{13}$ and this was reflected in the different prevalence of TET2 mutations between our cohort and the other two. Furthermore, for TET2 missense mutations, the missense mutations with unknown biologic significance were censored, which would possibly lead to lower frequency of TET2 mutations in this study. ${ }^{13}$ The ethnic difference might be another influencing factor. Recently, CSF3R mutation was found closely associated with CEBPA mutation in both adult and pediatric AML patients. ${ }^{14}$ Unfortunately, CSF3R mutation was not included in our panel.

According to the current ELN guidelines, allo-HSCT is not routinely recommended in $C E B P A^{\mathrm{dm}}$ patients in first $\mathrm{CR}$. Indeed, though postremission chemotherapy alone in first CR correlated with a significantly higher relapse rate and shorter DFS as compared with allo-HSCT, the high relapse rate in the chemotherapy subgroup did not translate into a significant inferior OS because relapsed patients still showed a high second $\mathrm{CR}$ rate. ${ }^{6,15}$ The relapse rate of $C E B P A^{\mathrm{dm}}$ patients after first $\mathrm{CR}$ in this study was $37.5 \%$ in total $C E B P A^{\mathrm{dm}}$ patients and $45.7 \%$ in the postremission chemotherapy subgroup, which was comparable with that reported previously $(36.2 \%$ $41 \%){ }^{1,6}$ Surprisingly, all WT1-mutated CEBPA ${ }^{\mathrm{dm}}$ patients, if not transplanted in first $C R$, encountered disease relapse (Table 1 ). The second CR rate was only $25 \%$ after re-induction, which was much lower than that (around $80 \%$ ) in the total CEBPA ${ }^{\mathrm{dm}}$ cohort. $^{6}$ Taken together, it is suggested that $C E B P A^{\mathrm{dm}}$ patients with WT1 co-mutation 
receive HSCT in first $\mathrm{CR}$ given the high relapse rate and gravid prognosis if relapse occurs. Further prospective randomized studies are warranted to validate the point.

This study clearly demonstrates the heterogeneous clinical outcome of $C E B P A^{\mathrm{dm}}$ patients and provides useful clinical information on refining the 2017 ELN risk categorization. Concomitant WT1 mutations suffice to be a marker for dismal prognosis in $C E B P A^{\mathrm{dm}}$ patients and help in our understanding of the process of leukemogenesis in this group. More importantly, allo-HSCT in first CR may be indicated for long-term disease control of this poorrisk entity.

Feng-Ming Tien, ${ }^{1,2,3}$ Hsin-An Hou, ${ }^{1}$ Jih-Luh Tang, ${ }^{1,3}$ Yuan-Yeh Kuo, ${ }^{4}$ Chien-Yuan Chen, ${ }^{1}$ Cheng-Hong Tsai, ${ }^{1,3}$ Ming Yao, ${ }^{1}$ Chien-Ting Lin, ${ }^{1,3}$ Chi-Cheng Li, ${ }^{1,3}$ Shang-Yi Huang, ${ }^{1}$ Bor-Sheng Ko, ${ }^{1}$ Szu-Chun Hsu, Shang-Ju Wu, Jia-Hau Liu, ${ }^{1,3}$ Sheng-Chieh Chou, ${ }^{1}$ Woei Tsay, ${ }^{1}$ Mei-Hsuan Tseng, ${ }^{1}$ Ming-Chih Liu, ${ }^{6}$ Chia-Wen Liu, ${ }^{6}$ Liang-In Lin, ${ }^{7}$ Wen-Chien Chou, and Hwei-Fang Tien

${ }^{1}$ Department of Internal Medicine, Division of Hematology, National Taiwan University Hospital; ${ }^{2}$ Graduate Institute of Clinical Medicine, College of Medicine, National Taiwan University; ${ }^{3}$ Tai-Cheng Stem Cell Therapy Center, National Taiwan University; ${ }^{4}$ Graduate Institute of Oncology, College of Medicine, National Taiwan University; Departments of Laboratory Medicine and ${ }^{6}$ Pathology, National Taiwan University Hospital and 'Department of Clinical Laboratory Sciences and Medical Biotechnology, College of Medicine, National Taiwan University, Taipei, Taiwan

Acknowledgments: we would like to acknowledge the service provided by the DNA Sequencing Core of the First Core Laboratory at National Taiwan University College of Medicine. We are greatly indebted to all the AML patients for contributing their samples and clinical data.

Correspondence:hsinanhou@ntu.edu.tw/hftien@ntu.edu.tw doi:10.3324/haematol.2018.189043

Information on authorship, contributions, and financial \& other disclosures was provided by the authors and is available with the online version of this article at www. haematologica.org.

\section{References}

1. Dufour A, Schneider F, Metzeler KH, et al. Acute myeloid leukemia with biallelic CEBPA gene mutations and normal karyotype repre- sents a distinct genetic entity associated with a favorable clinical outcome. J Clin Oncol. 2010;28(4):570-577.

2. Taskesen E, Bullinger L, Corbacioglu A, et al. Prognostic impact, concurrent genetic mutations, and gene expression features of AML with CEBPA mutations in a cohort of 1182 cytogenetically normal AML patients: further evidence for CEBPA double mutant AML as a distinctive disease entity. Blood. 2011;117(8):2469-2475.

3. Fasan A, Haferlach C, Alpermann T, et al. The role of different genetic subtypes of CEBPA mutated AML. Leukemia. 2014;28(4):794-803.

4. Hou HA, Lin CC, Chou WC, et al. Integration of cytogenetic and molecular alterations in risk stratification of 318 patients with de novo non-M3 acute myeloid leukemia. Leukemia. 2014;28(1):50-58.

5. Grossmann V, Haferlach C, Nadarajah N, et al. CEBPA doublemutated acute myeloid leukaemia harbours concomitant molecular mutations in $76.8 \%$ of cases with TET2 and GATA2 alterations impacting prognosis. Br J Haematol. 2013;161(5):649-658.

6. Schlenk RF, Taskesen E, van Norden Y, et al. The value of allogeneic and autologous hematopoietic stem cell transplantation in prognostically favorable acute myeloid leukemia with double mutant CEBPA. Blood. 2013;122(9):1576-1582.

7. Tsai CH, Hou HA, Tang JL, et al. Genetic alterations and their clinical implications in older patients with acute myeloid leukemia. Leukemia. 2016;30(7):1485-1492.

8. Hou HA, Liu CY, Kuo YY, et al. Splicing factor mutations predict poor prognosis in patients with de novo acute myeloid leukemia. Oncotarget. 2016;7(8):9084-9101.

9. Hou HA, Huang TC, Lin LI, et al. WT1 mutation in 470 adult patients with acute myeloid leukemia: stability during disease evolution and implication of its incorporation into a survival scoring system. Blood. 2010;115(25):5222-5231.

10. Virappane P, Gale R, Hills R, et al. Mutation of the Wilms' tumor 1 gene is a poor prognostic factor associated with chemotherapy resistance in normal karyotype acute myeloid leukemia: the United Kingdom Medical Research Council Adult Leukaemia Working Party. J Clin Oncol. 2008;26(33):5429-5435.

11. Papaemmanuil E, Gerstung M, Bullinger L, et al. Genomic classification and prognosis in acute myeloid leukemia. N Engl J Med. 2016; 374(23):2209-2221.

12. Theis F, Corbacioglu A, Gaidzik VI, et al. Clinical impact of GATA2 mutations in acute myeloid leukemia patients harboring CEBPA mutations: a study of the AML study group. Leukemia. 2016;30(11):2248-2250.

13. Chou WC, Chou SC, Liu CY, et al. TET2 mutation is an unfavorable prognostic factor in acute myeloid leukemia patients with intermediate-risk cytogenetics. Blood. 2011;118(14):3803-3810.

14. Lavallee VP, Krosl J, Lemieux S, et al. Chemo-genomic interrogation of CEBPA mutated AML reveals recurrent CSF3R mutations and subgroup sensitivity to JAK inhibitors. Blood. 2016;127(24):3054-3061.

15. Ahn JS, Kim JY, Kim HJ, et al. Normal karyotype acute myeloid leukemia patients with CEBPA double mutation have a favorable prognosis but no survival benefit from allogeneic stem cell transplant. Ann Hematol. 2016;95(2):301-310. 